\title{
ANÁLISIS DE MICROSATÉLITES EN CÉLULAS EXFOLIADAS DEL SEDIMENTO URINARIO. SU UTILIDAD PARA LA DETECCIÓN DEL CÁNCER VESICAL. ESTUDIO COMPARATIVO CON CITOLOGÍA URINARIA
}

\author{
R. MOLINA BURGOS*, J.M. MILLÁN SALVADOR**, J.S. OLTRA SOLER**, \\ J.F. JIMÉNEZ CRUZ*
}

*Servicio de Urología. **Unidad de Genética. Hospital Universitario La Fe. Valencia.

Actas Urol Esp. 27 (8): 618-628, 2003

\section{RESUMEN}

ANÁLISIS DE MICROSATÉLITES EN CÉLULAS EXFOLIADAS DEL SEDIMENTO URINARIO. SU UTILIDAD PARA LA DETECCIÓN DEL CÁNCER VESICAL. ESTUDIO COMPARATIVO CON CITOLOGÍA URINARIA

INTRODUCCIÓN: Teniendo en cuenta la precocidad de las alteraciones genéticas en la carcinogénesis de los tumores de vejiga, la valoración de estos cambios a nivel de 9p 21-22 por medio de marcadores microsatélites podrian ser útiles para el diagnóstico y seguimiento.

OBJETIVO: Evaluar el uso de marcadores microsatélites y la utilidad de pérdida de heterocigosidad (LOH) e inestabilidad de microsatélites (MSI) en células exfoliadas del sedimento urinario para él diagnostico de tumores vesicales.

MATERIAL Y MÉTODO: Amplificamos con PCR el DNA de muestras de orina y sangre de 160 pacientes con tumor vesical. Con 4 marcadores microsatélites de 9p 21-22 (D9S747-D9S171-D9S162-IFNA) y 1 de cromosoma 4 (D4S243) se analizó LOH/MSI en células del sedimento urinario. Utilizamos la citología urinaria como método comparativo y el análisis histológico del tejido obtenido por R.T.U, como diagnóstico de referencia. Calculamos la sensibilidad y especificidad del método y si existía alguna correlación con estadio y grado tumoral.

RESULTADOS: Se pudo utilizar correctamente 150 muestras. En 111 encontramos LOH/MSI (sensibilidad del 74\%). La citología pudo descubrir solamente 60 pacientes (sensibilidad 40\%). Encontramos mayor número de alteraciones microsatélites (AM) en tumores superficiales (sensibilidad $77,3 \%$ vs. $28,8 \%$ de la citologia) y valores estadísticamente significativos (MSI p<0,001 - LOH p<0,004) cuando comparamos tumores GI-II vs. GIII. El marcador con mayor sensibilidad fue D4S243 con un 40\%. Un paciente con AD. Carcinoma de próstata y otro con cistitis crónica dieron resultado falsos positivos.

CONCLUSIONES: El estudio de LOH/MSI en tumores vesicales con 5 marcadores microsatélites, según nuestros datos presenta una sensibilidad del $74 \%$.

El mayor números de LOH/MSI la encontramos en tumores superficiales y tumores GI-GII. Aunque no permite descartar la realización de cistoscopias en el diagnóstico y seguimiento, mejora la sensibilidad de la citología urinaria y seria una alternativa diagnostica como un procedimiento no invasivo. (Beca F.I.U).

PALABRAS CLAVE: Microsatélites. Pérdida de hererocigosidad (LOH). Inestabilidad (MSI). Cáncer de vejiga. Citologia.

\section{ABSTRACT}

MICROSATELLITE ANALYSIS IN EXFOLIATED CELLS FROM URINE SEDIMENT. UTILITY FOR BLADDER CANCER DETECTION. COMPARATIVE STUDY WITH URINARY CYTOLOGY

INTRODUCTION: Taking into account the precocity of the genetic alterations in the carcinogenesis of the bladder tumors, the valuation of these changes at a level of 9p 21-22 by means of microsatellite markers could be useful for the diagnostic and follow-up.

PURPOSE: To evaluate the use of microsatellite markers and the utility of loss of heterozigosity (LOH) and microsatellite instability (MSI) in exfoliated cells from urine sediment. This observation offers the possibility of tumor detection by examining the DNA of urinary sediment.

MATERIALS AND METHODS: We amplified with PCR the DNA of urine and blood samples from 160 patients with bladder cancer. We analysed LOH/MSI in cells from urinary sediment using four microsatellite markers of 9p 21-22 (D9S747-D9S171-D9S162-IFNA) and one from chromosome 4 (D4S243). The urinary cytology was used as comparative method and histological examination of tissue obtained by transurethral resection (TUR) as reference diagnostic. We calculated the sensibility and specificity of this method and if there was some correlation between stage and grade tumoral.

RESULTS: We could use 150 samples correctly. In 111 samples we found LOH/MSI (sensibility $74 \%$ ). The cytology was positive only in 60 patients (sensibility 40\%). We found a bigger number of microsatellite alterations (AM) in superficial tumors (sensibility $77,3 \%$ vs. $28,8 \%$ for the cytology) and these were significant when comparing tumors GI-II vs. GIII (MSI $p<0,001-$ LOH $p<0,004$ ). The marker with more sensibility was D4S243 with $40 \%$. One patient with prostate carcinoma and another one with chronic cystitis gave false positive results.

CONCLUSIONS: The study of LOH/MSI in bladder tumors with 5 microsatellites markers, according to our results showed a sensibility of $74 \%$.

The biggest number in LOH/MSI was found in superficial tumors and GI-GII tumors. Although we cannot discard the cystoscopy study in the diagnostic and follow-up, the sensibility of the urine cytology is better and could be one alternative diagnostic as a non-invasive procedure.

KEY WORDS: Microsatellite. Loss of heterozigosity (LOH). Inestability (MSI). Bladder cancer. Cytology. 
$\mathrm{E}^{\mathrm{t}}$ carcinoma de vejiga representa unos de los tumores más frecuentes de la economía.

Es la cuarta neoplasia más frecuente en el hombre y octavo en la mujer en el mundo occidental, con una relación de 4:1 a favor del hombre y cerca de 54.000 nuevos casos diagnosticados durante 2001 en los EE.UU ${ }^{1}$. En España las cifras actuales muestran un incremento en las últimas décadas. En 1986 se diagnosticaban 19,5 casos cada $100.000 \mathrm{~h}$, cifra que actualmente se eleva a 27 casos nuevos cada 100.000 h. ${ }^{2}$.

Una característica importante de estos tumores es el alto porcentaje de recidivas que presenta, entre un 70 a $75 \%$, mientras que un 10-15\% progresan a estadios más avanzados ${ }^{3,4}$.

Esto requiere que los pacientes con tumores vesicales, estén en permanente seguimiento para descubrir nuevas recidivas después del tratamiento inicial.

A pesar de que en los últimos años se han evaluado diferentes métodos para la detección y seguimiento de estos tumores, la citología y la cistoscopia sigue siendo lo más adecuado para él diagnostico de la recidiva tumoral.

La citología urinaria fue la primera técnica utilizada para detectar la presencia de células tumorales en el sedimento urinario ${ }^{5}$. Su especificidad elevada favorece su uso en la practica diaria pero su baja sensibilidad, sobre todo en tumores de bajo grado, no le permite ser el método ideal $^{6}$. Mientras que la cistoscopia, utilizada cada 3-4 meses durante los 2 primeros años después de la resección inicial, presenta el inconveniente de ser una técnica invasiva, y no exenta de morbilidad, a pesar de que hoy en día disponemos de instrumental mejor tolerado por los pacientes.

Existen otros métodos como la citometría de flujo, P53 en orina, telomerasa, producto de degradación del fibrinógeno, utilizados experimentalmente con resultados dispares ${ }^{7}$.

La FDA ha aprobado el uso de pruebas como el BTA y la determinación de la proteína de la matriz nuclear (NMP22) en orina pero no reconocen los carcinomas con tanta exactitud que permitan cambiar los regímenes cistoscópicos actuales. Todavía no se ha podido desarrollar un método sencillo, fiable y con elevado porcentaje de sensibilidad que pueda reemplazar a la cistoscopia y citología ${ }^{8}$.
Los avances ocurridos en el campo de la biología molecular han permitido descubrir regiones denominadas microsatélites, que son secuencias de DNA formadas por repeticiones en tándem de di, tri, o tetranucleótidos que se localizan a lo largo de todo el genoma humano y son altamente polimórficas ${ }^{9,10}$. Hay diferentes trabajos que han confirmado que secuencias microsatélites de determinadas regiones presentan pérdida de heterocigosidad (LOH) y/o inestabilidad (MSI) en diversos tumores ${ }^{11}$.

La inactivación de genes supresores de tumor es la principal causa de la tumorogénesis y la perdida de los segmentos cromosómicos que contienen estos genes es uno de los mecanismos de esta inactivación. Por lo tanto, el estudio de la LOH de secuencias microsatélites localizadas en las regiones cercanas a estos genes supresores es una herramienta útil, rápida y de bajo coste para el diagnóstico de tumores. Otro mecanismo implicado en la evolución tumoral son mutaciones como consecuencia de un defecto en el sistema de reparación del DNA. Estas mutaciones pueden descubrirse por análisis de inestabilidad de microsatélites $(M S I)^{12,13}$.

En varios trabajos se han utilizado las alteraciones de microsatélites (LOH e MSI) para descubrir la presencia de células neoplásicas en diferentes fluidos orgánicos como plasma, suero, esputo y orina ${ }^{14-17}$.

Mao et al. y Steiner et al., en los estudios de referencia sobre el tema, identificaron cáncer vesical con marcadores microsatélites analizando DNA extraído de células tumorales presentes en el sedimento urinario ${ }^{13,18}$.

La existencia de MSI en células tumorales del sedimento urinario es controvertida desde que se observo en algunos estudios y en otros no ${ }^{19-21}$.

Se sabe actualmente que el cromosoma 9p se encuentra involucrado en los primeros pasos de la tumorogénesis del cáncer vesical y que el mayor número LOH en 9p estaría mas relacionada con tumores superficiales que con tumores infiltrantes ${ }^{19,22}$.

La LOH en cromosoma $11 \mathrm{p}, 3 \mathrm{p}, 13 \mathrm{p}, \mathrm{y} 17 \mathrm{p}$ aparece asociado a tumores infiltrantes, mientras que existiría una asociación estadísticamente significativa entre grado tumoral /estadio para cromosoma 8 y cromosoma $4^{23-28}$. 
Teniendo en cuenta la precocidad de las alteraciones genéticas en la carcinogénesis de los tumores vesicales, sobre todo $\mathrm{LOH}$ de la región 9p21-22p en donde probablemente se localizaría un gen supresor de tumor implicado en el cáncer vesical, la valoración de estos cambios podrían ser útil para él diagnostico y seguimiento ${ }^{29}$.

En el presente estudio investigamos la viabilidad del análisis de microsatélites (LOH e MSI) para detectar la presencia de células neoplásicas en el sedimento urinario, utilizando un tablero de 5 marcadores microsatélites que permitan analizar estos cambios a nivel de 9p21-22 y cromosoma 4, además realizar análisis genético en tejido tumoral y comparar con los hallazgos en orina. Por otra parte buscar si existe una correlación entre LOH e MSI con estadio y grado tumoral.

Utilizamos la citología urinaria como método comparativo y el análisis histológico del tejido obtenido por R.T.U, como diagnostico de referencia.

\section{MATERIAL Y MÉTODO}

PACIENTES: Se estudiaron muestras de sangre y orina de 160 pacientes ingresados en el Servicio de Urología del Hospital Universitario "La $\mathrm{Fe}$ " de Valencia, España, para tratamiento quirúrgico endoscópico por tumor vesical. Las muestras se recolectaron entre noviembre de 2000 y enero de 2002. El grupo estaba representado por 131 hombres y 29 mujeres con edades comprendidas entre 47-87 años, con 91 pacientes con tumores primarios, 60 con tumores recidivado y 9 pacientes con sospecha de persistencia tumoral luego de tratamiento con radio-quimioterapia.

El grupo control lo constituian 40 pacientes (30 hombres y 10 mujeres) sin antecedentes de cáncer de vejiga con edades muy similares al grupo de estudio. A todos los pacientes que constituían el grupo control se le realizo cistoscopia ya que eran pacientes ingresados para RTU de Próstata y mujeres con Incontinencia urinaria de esfuerzo para cirugía.

MUESTRAS DE ORINA, SANGRE Y TUMOR: Obtuvimos previo a la RTU de vejiga dos muestras de orina de $50 \mathrm{ml}$ a primera hora de la mañana por micción espontánea para estudio genético y citológico (PAP). Además se obtuvo una muestra de sangre de $20 \mathrm{ml}$ que era colocada en un tubo con EDTAk3 como anticoagulante. Una de las muestras de orina se enviaba al servicio de patología para realizar citología urinaria (PAP) según técnica habitual, sin hacer referencia sobre el motivo del pedido para evitar los sesgos en la interpretación de los resultados. Tanto la muestra de orina y sangre para estudio genético se conservaban a $4^{\circ} \mathrm{C}$ hasta su procesamiento 6 hs después aproximadamente.

Durante la intervención quirúrgica se realizo biopsia tumoral para análisis molecular. Se hizo hincapié en tomar muestra sin la contaminación del tejido normal. Inmediatamente eran congeladas a $-70^{\circ}$ hasta la extracción del DNA.

EXTRACCIÓN DE DNA: Orina: La muestra se centrifugaba durante 10' a 2000 rpm, se decantaba el sobrenadante para posteriormente realizar dos lavados con solución tampón. Se añade

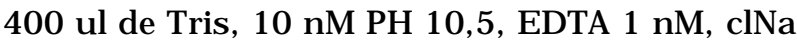
0,15 m., agregando luego SDS al 10\% y $10 \mathrm{ul} \mathrm{de}$ proteinasa K. Se encubaba a $56^{\circ} \mathrm{C}$ durante $24 \mathrm{hs}$ y posterior purificación con fenol/cloroformo.

Tumor: Se realizaba mínimos cortes de tejido congelado en medio de cultivo y lavado posterior con solución tampón y se continuaba con la misma técnica de extracción que para la orina.

Sangre: La extracción del DNA genómico constitucional se realizo según el método clásico de digestión enzimático con proteinasa $\mathrm{K}$ y precipitación con fenol/cloroformo.

Luego de la extracción todas, las muestras eran congeladas a $-20^{\circ}$ hasta su amplificación con PCR. (Reacción de la cadena de la polimerasa).

MICROSATÉLITES Y AMPLIFICACIÓN: Utilizamos 4 marcadores microsatélites localizados en el interior o vecindad de regiones con frecuentes dilecciones o perdidas en el cromosoma 9p21-22, (D9S747 (5' gccattattgactctgaaaagac-3'- 5'caggctctctaaaatatgaacaaaat-3') D9S171 (5' accetagcactgatggtatagtct-3'-5'gggcaaggagagactct-3') D9S162 (5' atgtggttagggttettcagag-3'-5' cccacaacaaateteetcac-3') IFNA (5' gtaaggtggaaacccccact-3'-5'tgcgcgttaagttaagttaattggtt-3') y 1 marcador que nos permitiera estudiar inestabilidad en cromosoma 4, D4S243 (5' gagcctgtgatcctgttggtg-3'-5'gtctctettteteettgcag-3'). 
La reacción de la cadena de la polimerasa (PCR) se utilizó para la amplificación del DNA con cada uno de los marcadores. El programa consistía en una desnaturalización inicial a $95^{\circ} \mathrm{C}$ durante 5 minutos, 35 ciclos de $\left[95^{\circ} \mathrm{C} 30 \mathrm{seg}-\right.$ $55^{\circ} \mathrm{C} 30 \mathrm{seg}-72^{\circ} \mathrm{C}$ durante 10 minutos] y un paso de elongación final a $72^{\circ} \mathrm{C}$ durante $10 \mathrm{minu}$ tos. Posteriormente se modifico con los marcadores D9S747 y D9S171 a la temperatura de unión con los cebadores de 55 a $60^{\circ} \mathrm{C}$.

El producto de PCR se sometió a electroforesis en un gel de agarosa al $1 \%$ para comprobar la eficacia de la reacción.

Por último se realizaron geles de poliacrilamida al $12 \%$ de $40 \times 50 \mathrm{~cm}$. La electroforesis se lleva a cabo a 1600 voltios durante 190 minutos y la detección del producto de PCR se realizó por tinción de plata.

VALORACIÓN DE LOH e MSI: Tanto la LOH como la MSI se detectaban al comparar el patrón de alelos presente en el DNA constitucional y el DNA detectado en orina y/o tumor. Cuando con un determinado marcador desaparece por completo unos de los alelos en el DNA de orina y/o tumor se considera $\mathrm{LOH}$ (Fig. 1a). También se considero como LOH un descenso de hasta el $50 \%$ en la intensidad de las bandas alélicas tanto de orina como de tumor (Fig. 1b). Mientras que si visualizábamos una nueva banda en las muestras de orina y/o tumor con respecto a DNA constitucional considerábamos MSI (Fig. 1c).

Para evitar sesgos en la lectura de los resultados los geles una vez teñidos y secados, fueron leídos por 2 dos observadores expertos del laboratorio de Genética Molecular.

El análisis molecular de las muestras se llevó a cabo sin el conocimiento de datos clínico y patológico de los pacientes.

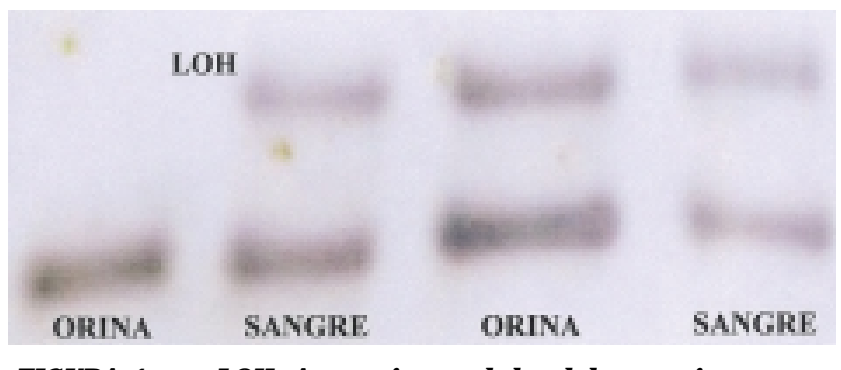

FIGURA 1a: LOH: Ausencia total de alelo en orina. ESTUDIO NORMAL: 2 alelos en orina/sangre.

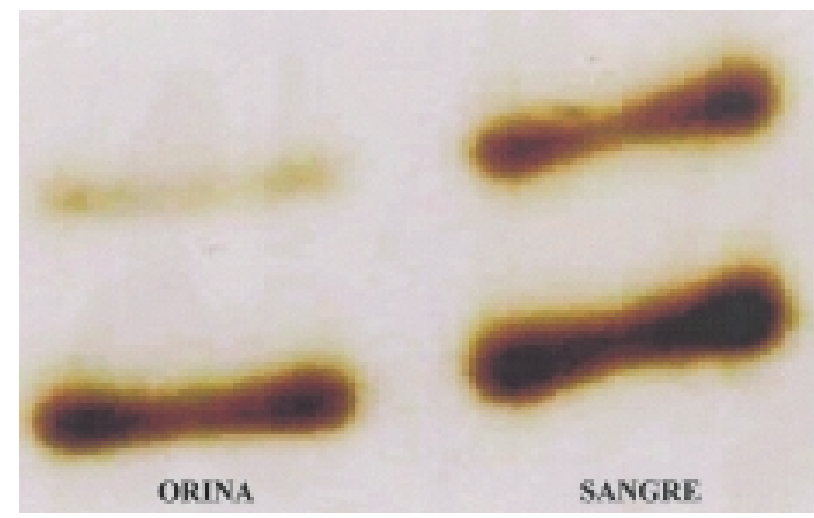

FIGURA 1b: LOH: < del 50\% de intensidad en unos de los alelos de orina.

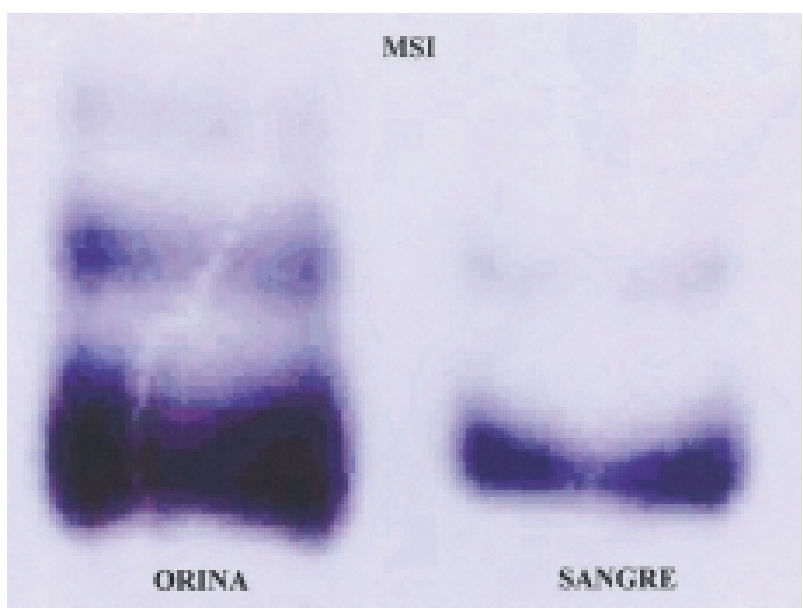

FIGURA 1c: MSI: Nuevo alelo en orina.

ANÁLISIS ESTADÍSTICO: Utilizamos el programa estadístico SPSS 10.0 (Chicago, IL) para el análisis de todos los datos. La sensibilidad (capacidad de una prueba de detectar pacientes enfermos) y especificidad (capacidad de una prueba para detectar pacientes sanos) fue calculada tanto para el análisis genético como para la citología en forma global, por estadio, grado de diferenciación

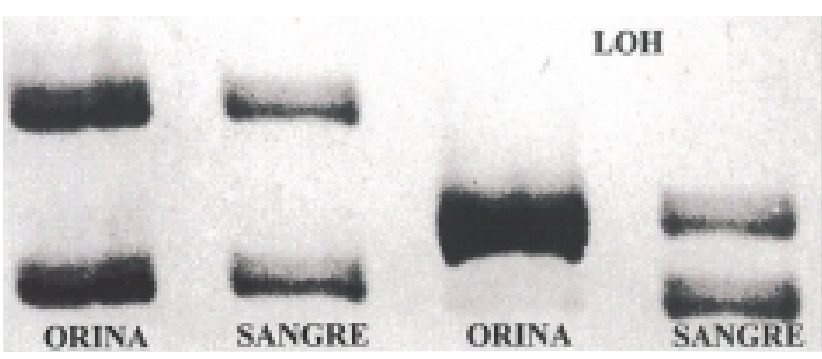


celular y por marcador microsatélite. Además evaluamos asociaciones entre diferentes variables usando las pruebas de chi cuadrado, test de tendencia y prueba de Man-Whitney.

\section{RESULTADOS}

Analizamos muestras de sangre y orina de 160 pacientes con diagnóstico de tumor vesical. El análisis genético lo realizamos con un tablero de 5 marcadores microsatélites que comparaban DNA en orina, tejido tumoral y sangre periférica. La extracción de DNA en orina se logro realizar en 152 pacientes. En los 8 restantes no se pudo obtener suficiente material para el estudio, lo cuál lo podríamos relacionar con el aprendizaje de la técnica de extracción de DNA en orina o bien al escaso volumen de orina disponible. El DNA tumoral se amplifico correctamente en 45 muestras. La presencia de tejido normal, "contaminando" la muestra sobre todo en tumores pequeños y superficiales dificultaba su interpretación.

Utilizamos el TNM de la UICC de 1997 para la clasificación anatomopatológica encontrando en el grupo en estudio 25 tumores Ta, 46 Tla y 26 $\mathrm{T} 1 \mathrm{~b}$, con un total de 97 tumores superficiales con un porcentaje del $64,6 \%$. Mientras que los tumores infiltrantes fueron 53 , con 30 en estadio T2a, 15 T2b y 8 T3-T4 y que representaban el 35,3\%.

El grado de diferenciación celular estaba dado por 53 pacientes con tumores GI, 58 con GII y 39 pacientes con tumores GIII. El porcentaje fue del $35,3 \%, 38,6 \%$ y $26 \%$ respectivamente.

El total de muestras analizadas fueron 152. Dos pacientes dieron como resultados falsos positivos. Se trataba de un paciente con diagnostico de cistitis crónica y el restante con adenocarcinoma de próstata con infiltración vesical que demostró LOH e MSI con los 5 marcadores.

De los 150 pacientes con diagnostico anatomopatológico de carcinoma de células transicionales (CCT) en 111 muestras encontramos $\mathrm{LOH}$ o MSI con algunos de los 5 marcadores utilizados, mientras que con al citología descubrimos tumor solamente en 60 pacientes. La sensibilidad global del análisis genético en el sedimento urinario en pacientes portadores de cáncer vesical fue del $74 \%$, siendo para la citología en este mismo grupo de pacientes del $40 \%$.
Al analizar la sensibilidad por estadio en los tumores superficiales observamos que en los TaTla-b la sensibilidad del test permitía diagnosticar la presencia de tumor en el $72 \%, 80,4 \%$ y $77 \%$ de estos pacientes, siendo del $77,3 \%$ la sensibilidad global para todos los tumores superficiales. En estos la citología habría diagnosticado el $8 \%$, $34 \%$ y el $38,4 \%$ de los pacientes con tumores superficiales. El porcentaje global para el estudio citológico en este grupo de tumores fue del $28,8 \%$ (Tabla I).

TABLA I

TUMORES SUPERFICIALES (Ta - T1a - T1b)

\begin{tabular}{|c|c|c|c|}
\hline Estadio & Muestras & Pérdidas & Citología (+) \\
\hline Ta & 25 & 18 (S 72\%) & 2 (S 8\%) \\
\hline T1a & 46 & 37 (S 80,4\%) & 16 (S 34\%) \\
\hline T1b & 26 & 20 (S 77\%) & 10 (S 38,4\%) \\
\hline TOTAL & 97 & 75 (S 77,3\%) & $28(28,8 \%)$ \\
\hline
\end{tabular}

S: Sensibilidad.

En los tumores infiltrantes la prueba pudo identificar 36 tumores de 53, lo que indica una sensibilidad del $67,9 \%$, no muy significativa con respecto a la citología que pudo encontrar 33 (sensibilidad del 62,2\%).

La sensibilidad por grado de diferenciación celular estuvo dada por un $73,5 \%$ para los tumores GI y 74,1\% para los GII. En los GIII la citología tuvo mayor sensibilidad con un porcentaje del $82 \%$ frente al $69,2 \%$ del análisis molecular (Tabla II).

De la serie, 13 pacientes eran tumores Ta GI de los cuales 7 presentaban LOH o MSI con algunos de los marcadores. El estudio citológico fue negativo en 6 de ellos.

TABLA II

GRADO DE DIFERENCIACIÓN CELULAR (GI-GII-GIII)

\begin{tabular}{|c|c|c|c|}
\hline Grado & Muestras & Pérdidas & Citología (+) \\
\hline G1 & 53 & 40 (S 75,4\%) & 5 (S 9,4\%) \\
\hline GII & 58 & 44 (S 75,8\%) & 23 (S 39,6\%) \\
\hline GIII & 39 & 27 (S 69,2\%) & 32 (S 82\%) \\
\hline TOTAL & 150 & 111 (S 74\%) & 60 (S 40\%) \\
\hline
\end{tabular}

S: Sensibilidad. 
Contrariamente 7 pacientes eran T2b GIII. En 4 de ellos encontramos alteraciones genéticas a través del análisis de microsatélites, pero la citología pudo diagnosticar los 7 ya que todos presentaban PAP sospechoso o positivo.

En 11 pacientes del grupo estudiado, de los cuales 6 eran tumores superficiales y 5 infiltrantes no se demostró alteraciones genéticas ( $\mathrm{LOH}$ e MSI) en orina, pero el PAP Urinario fue sospechoso. Mientras que en 4 muestras, todos infiltrantes, el PAP fue positivo pero no se hallaron alteraciones genéticas en el sedimento urinario (Tabla III).

Al analizar la sensibilidad de cada marcador microsatélite encontramos que el marcador D4S243 presenta la más alta sensibilidad, 40\%. Esto es debido que con este marcador microsatélites detectamos el mayor número de muestras con MSI, que sumados a las LOH encontradas con el mismo hacen un total de 60 (Tabla IV).

De estas 40 muestras con MSI en cromosoma 4, 30 pacientes tenían tumores superficiales, Ta 7; T1a 19; T1b 4; los restantes eran infiltrantes. En 30 muestras presentaban además LOH con otros marcadores.

Los pacientes con tumores superficiales e MSI en cromosoma 4 presentaban citología urinaria negativa en 6 de 7 pacientes en estadio Ta, 14 de 19 en Tla y 2 de 4 en T1b. Existe por lo tanto una

\section{TABLA III}

LOH/MSI - FALSOS NEGATIVOS

\begin{tabular}{|c|c|c|c|}
\hline Muestras & Citología & Marcadores & Tumores \\
\hline 11 & Sospechosa & Negativos & $\begin{array}{c}\text { T1a 4; T1b 2; } \\
\text { T2a 3; T2b 1; T3 1 }\end{array}$ \\
\hline 4 & Positivo & Negativo & T2b 4 \\
\hline
\end{tabular}

\section{TABLA IV}

SENSIBILIDAD POR MARCADORES

\begin{tabular}{||c|c|c|c||}
\hline Marcadores & Pérdidas & Normal & Sensibilidad \\
\hline D9S747 & 43 & 107 & $28 \%$ \\
\hline D9S171 & 39 & 111 & $26 \%$ \\
\hline D9S162 & 40 & 110 & $26,6 \%$ \\
\hline IFNA & 46 & 104 & $30,6 \%$ \\
\hline D4S243 & 60 & 90 & $40 \%$ \\
\hline
\end{tabular}

asociación estadísticamente significativa $(p=0,002)$ entre tumores superficiales, por la presencia de MSI en cromosoma 4, con relación a tumores infiltrantes.

La presencia de MSI y LOH en tumores GI-II con relación a GIII también es estadísticamente significativa, $\mathrm{p}<0,001$ para MSI y $\mathrm{p}<0,004$ para $\mathrm{LOH}$ respectivamente. Un solo paciente presenta MSI con un marcador diferente al cromosoma 4, el D9S162 para la muestra nº 95.

Analizamos también, como variables independientes, la posibilidad de que existan valores estadísticamente significativos entre el hallazgo de $\mathrm{LOH}$ en tumores Ta con relación a T1a-b. Solo encontramos resultados ligeramente significati$\operatorname{vos}(\mathrm{p}<0,04)$. No encontramos valores estadísticamente significativos en tumores infiltrantes con respecto a LOH e MSI ( $\mathrm{p}>0,05)$, ni tampoco si analizamos grado de diferenciación celular con respecto a $\mathrm{LOH}$ e MSI.

De las 45 muestras de tumor que se analizaron encontramos en la mayoría de los casos idénticas alteraciones genéticas que en el análisis molecular del sedimento urinario (Fig. 2). En 6 muestras se identifican LOH y en 1 MSI que no eran perceptibles en las muestras de tumor. (Fig. 3a y b).

La especificidad del método fue del 100\%, ya que fue estudiado con un grupo control de 40 pacientes a quienes se le realizo igual método de extracción de DNA en orina y sangre. El DNA se amplificó con los mismos marcadores utilizados en el grupo en estudio. En ninguna de las muestras de estos pacientes encontramos alteraciones genéticas. A todos se le realizo cistoscopia durante la R.T.U. de próstata o la cervicopexia por

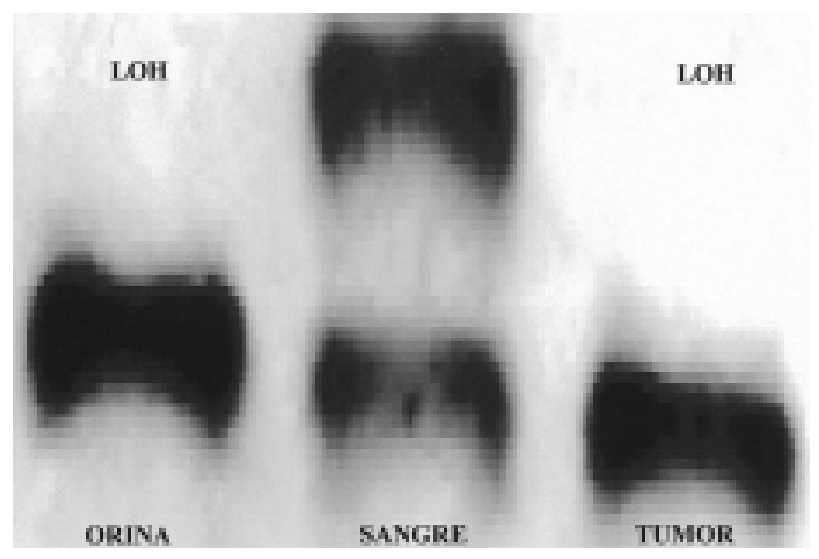

FIGURA 2. LOH: Ausencia de alelo en orina y tumor. 


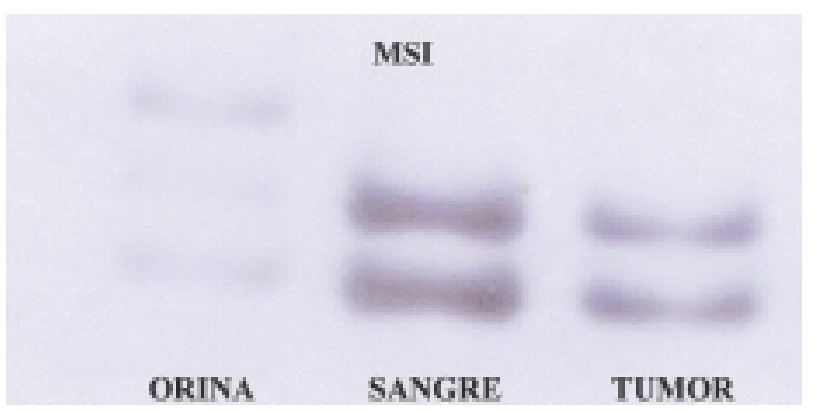

FIGURA 3a. MSI: Presencia de nuevo alelo en orina con sangre y tumor normales.

incontinencia de orina sin encontrar lesiones que hicieran sospechar la presencia de lesiones tumorales.

\section{DISCUSIÓN}

La citologia urinaria y la cistoscopia son actualmente los métodos utilizados para el diagnóstico y seguimiento de los tumores vesicales. Sin embargo, la citología presenta inconvenientes a la hora de diagnosticar tumores de bajo grado y estadio. A pesar del empeño puesto de manifiesto por los citopatólogos en identificar características que faciliten la distinción entre células normales y malignas en tumores de bajo grado, los valores de sensibilidad no superan el $25 \%{ }^{30}$.

Pese a que algunos autores describen ausencias de algunas alteraciones de los microsatélites en sus series de tumores de vejiga ${ }^{21}$ la mayoría señalan alteraciones genómicas como la pérdida de heterocigosidad (LOH)/inestabilidad (MSI) que se ha documentado en varios tipos de tumores incluyendo el cáncer de vejiga ${ }^{31-40}$.

En el cáncer de vejiga se han descrito alteraciones genómicas, tanto $\mathrm{LOH}$ como $\mathrm{MSI}$ en varias regiones cromosómicas concretas, 9p, 9q, 4, 8p, $11 \mathrm{p}, 17 \mathrm{p}, 18^{13,18,23,26}$.

Estas alteraciones de los marcadores microsatélites de determinadas regiones cromosómicas asociadas a tumores vesicales han permitido el diagnóstico de los mismos en porcentajes más elevados que la tradicional citología urinaria utilizando para el estudio molecular las células tumorales presente en el sedimento urinario ${ }^{13,18}$.

Dentro de las alteraciones descritas asociadas al cáncer de vejiga una de las más importantes por su frecuencia y precocidad es la $\mathrm{LOH}$ de la región 9p21.

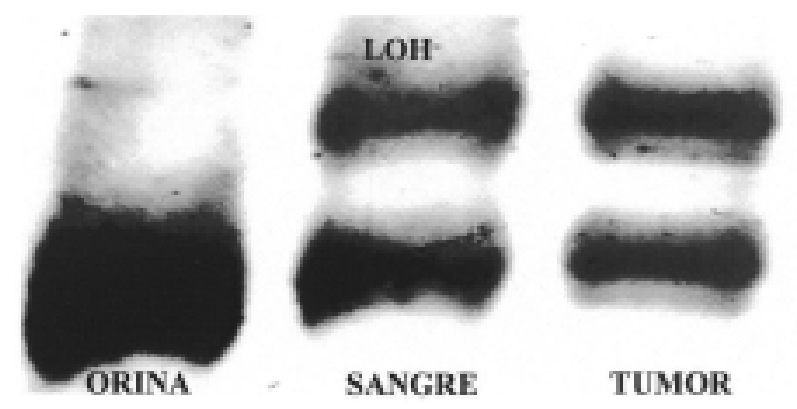

FIGURA 3b: LOH: Ausencia de alelo en orina con sangre $y$ tumor normales.

En el presente trabajo utilizando 4 marcadores de 9p2 1 (IFNA, D9S162, D9S171 y D9S747) y un marcador del cromosoma 4 (D4S243) en 150 muestras de orina y sangre de cada paciente, obteniendo una sensibilidad total, con los 5 marcadores de un $74 \%$ frente al $40 \%$ de la citología.

Esta sensibilidad es menor a la obtenida por Mao et al. ${ }^{13}$ en un estudio comparativo con citología utilizando 13 marcadores microsatélites, quienes detectaron $\mathrm{LOH}$ en el $95 \%$ de las muestras $(19 / 20)$ contra el $50 \%$ de la citología. Steiner et al. ${ }^{18}$ encontraron $\mathrm{LOH}$ en 10 de 11 pacientes empleando 20 marcadores microsatélites. Sepira et al. ${ }^{41}$ y Neves et al. ${ }^{42}$ también obtuvieron sensibilidades superiores al 95\% en sus series, mientras que con la citología urinaria lograron sensibilidades del 30-70\%.

Sourvinos et al. ${ }^{43}$ demostraron resultados del 93\% (26 de 28 casos) utilizando 15 microsatélites de las regiones donde se localizan p53 (17p), p16 (9p) y RB1 (13q). También Mourah ${ }^{20}$ demostró un $83 \%$ de sensibilidad y $100 \%$ de especificidad en un estudio con 31 pacientes con cáncer de vejiga.

Berger et al. ${ }^{44}$ detecta alteraciones microsatélites en 27 de 31 pacientes con panel de 17 marcadores. Igual número de marcadores utiliza von Knobloch R. et al. ${ }^{45}$ para identificar alteraciones de microsatélites $(A M)$ en suero, tumor y orina de 33,27 y 28 pacientes respectivamente en una serie de 39. Cuándo este autor utiliza los 5 marcadores más representativos de su panel, que involucran las regiones $8 \mathrm{p}$ y $9 \mathrm{p}$, la sensibilidad desciende al $61,5 \%$.

Valores similares a los obtenido por nuestro grupo fueron publicados por Utting et al. ${ }^{46}$ utilizando 6 marcadores microsatélites detectando un $75 \%$ de pacientes con AM en un total de 44 
tumores de vejiga. Anteriormente Utting et al. ${ }^{47}$ encuentra una sensibilidad $45-65 \%$ con 2 marcadores de 9 p en 16 pacientes con tumores de vejiga. También utilizando 6 marcadores microsatélites Selli et al. ${ }^{12}$ comunican valores de sensibilidad del $46 \%$ frente al $30 \%$ de la citología.

Existe en nuestro trabajo una marcada diferencia entre los valores de sensibilidad entre uno y otro método claramente demostrado sobre todo en tumores superficiales donde los valores diferenciales son significativos a favor del análisis de microsatélites en orina (Tabla V).

\section{TABLA V}

SENSIBILIDAD AM vs. CITOLOGÍA

\begin{tabular}{|l|c|c|}
\hline Tumores & LOH/ MSI & Citología \\
\hline Superficiales & S $77,3 \%$ & S $28,8 \%$ \\
\hline Infiltrantes & S $67,9 \%$ & S $62,2 \%$ \\
\hline
\end{tabular}

S: Sensibilidad/AM: Análisis de Microsatélites

Estos resultados demuestran una vez mas la escasa resolución de la citología frente a tumores de bajo grado y estadio, y el aporte que pueden realizar las técnicas de biología molecular al estudio, diagnostico y seguimiento de estos tumores.

El porcentaje de sensibilidad obtenida por nosotros puede deberse a:

a) El mayor tamaño de la muestra, no existen muchos trabajos previos con una serie que involucre este número de pacientes, sobre todo el número de tumores superficiales analizados.

b) El uso de 5 marcadores de solo dos regiones distintas. Es muy posible que al utilizar otros marcadores de estas regiones hubiese dado lugar a un porcentaje mayor de $\mathrm{LOH}$ y/o MSI.

c) Una tercera razón puede estar dada por la ausencia de células tumorales en orina debido al modo de obtención de las muestras, al analizar orina de micción espontánea matinal y no de lavado vesical, y por otro, a la contaminación por leucocitos.

La presencia de leucocitos puede enmascarar la LOH/MSI por predominio de células inflamatorias sobre las células tumorales. Van Rhif et al. ${ }^{48}$ sugiere en su trabajo la imposibilidad de realizar esta técnica ante la presencia de más de 20 leucocitos por campo microscópico en orina.
Estos resultados demuestran que el estudio de células tumorales presentes en el sedimento urinario de pacientes portadores de T. Vesicales con técnicas de biología molecular y utilizando un panel de marcadores que involucren preferentemente la región 9p21, permite elevar el porcentaje de sensibilidad con respecto a las técnicas tradicionalmente utilizadas.

Globalmente nuestros resultados así lo comprueban, sobre todo en tumores de estadio más bajo donde el porcentaje de sensibilidad obtenido fue del 80,4 en tumores Tla. En la bibliografia encontramos valores similares, $75 \%$ de sensibilidad en tumores superficiales utilizando igual números de marcadores pero en series más reducidas $^{46}$.

Respecto a la sensibilidad por marcadores, aunque D4S243 fue el más sensible con un 40\% todos los marcadores utilizados tenían porcentajes de alrededor del $30 \%$ de sensibilidad. Analizando los resultados obtenidos con los marcadores de 9p la sensibilidad estaría entre un 50$55 \%$, muy similar a las informadas por otros autores ${ }^{9}$.

Por otro lado la presencia en esta serie de mayor número de AM en tumores superficiales indica que aunque las alteraciones de distintas regiones están implicadas en el proceso de tumorogénesis del cáncer vesical, las regiones 9p y 4 son particularmente importantes en este proceso, y que están alteradas en un porcentaje mayor en tumores superficiales.

Con respecto al estadio tumoral, hemos encontrado una correlación estadísticamente significativa entre la presencia de alteraciones en microsatélites (AM), dada sobre todo por el hallazgo de MSI, y los tumores superficiales $(p=0,002)$. La presencia de AM en tumores de grado bajo (GI-II) es estadísticamente significativa tanto por el hallazgo de MSI $(\mathrm{p}<0,001)$ como por la presencia de $\mathrm{LOH}(\mathrm{p}<0,004)$.

Así pues, en conjunto se observa AM significativas con marcadores de $9 \mathrm{p}$ y 4 en tumores superficiales sobre todo, en tumores GI-GII.

Mao et al. ${ }^{13}$ y Berger et al. ${ }^{44}$ no encontraron AM en tumores GI con un panel de 17 microsatélites. Estos autores sugieren que los tumores GI no son sensibles a las alteraciones microsatélites, sin embargo, nuestros resultados contradicen 
sus hallazgos. Así mismo, Berger et al. ${ }^{44}$ obtienen mayor número de $\mathrm{LOH}$ en tumores infiltrantes que en superficiales, y mayor porcentaje de $\mathrm{LOH}$ en tumores de grado II-III que GI, es decir, al contrario que nosotros pero solamente describe un $2 \%$ de MSI en su serie.

Von Knobloch et al. ${ }^{45}$ no encuentran una relación significativa entre la presencia de AM, en suero y orina, con el estadio y grado tumoral.

Por el contrario, Habuchi et al. ${ }^{22}$ informan que por lo menos el $50 \%$ de los tumores de bajo grado y estadio han perdido segmentos en 9p. Christensen et al. ${ }^{19}$ describen al igual que nosotros una relación entre la AM en 9p y tumores de bajo grado mientras que las AM en marcadores de 2p, 17p, 9q, 5q y 10p se asociaban a tumores de alto grado y propone dos hipótesis para el desarrollo del cáncer de vejiga.

La primera estaría basada en una selección clonal $^{49}$ en la que en la fase infiltrante, los clones tumorales que presentan pérdida de $5 \mathrm{q}, 10 \mathrm{p}, 17 \mathrm{p}$ etc tienen ventaja en cuanto al crecimiento respecto a los clones que llevan la $\mathrm{LOH}$ de $9 \mathrm{p}$ de manera que en los Ta se observará la LOH de 9p mientras que en los infiltrantes las células con $\mathrm{LOH}$ en 9p van muriendo mientras crecen las que llevan el resto de alteraciones que son las que se detectarán. Una segunda hipótesis es que existan 2 vías hacia el estado infiltrante del tumor. Una vía que parte de tumores Ta y otra de carcinomas "in situ".

En cualquier caso, nuestros datos coinciden con Christiansen et al. ${ }^{19}$ por lo que respecta a AM de 9p y encontramos una relación clara entre la LOH de los marcadores de 9p y la presencia de tumores superficiales.

En 7 de 45 muestras de tumor las alteraciones genéticas encontradas fueron diferentes a las del sedimento urinario. Esto podría ser debido a la presencia de gran cantidad de células normales contaminando la muestra tumoral, sobre todo en tumores pequeños y superficiales, unas de las razones por la cuál discontinuamos el estudio, o bien, por la presencia de células en orina de un clon diferente al obtenido en el tejido tumoral analizado.

Mao et al. ${ }^{13}$ describió en la orina de dos pacientes AM que no estaban presentes en el tejido tumoral correspondiente.
El hallazgo de citología positiva o sospechosa sin AM en la muestra de orina (falsos negativos), estaría dado por la presencia de clones celulares que este grupo de marcadores microsatélites utilizados no serían capaces de detectar. Podríamos atribuirle también al elevado porcentaje de falsos positivos que presenta la citología urinaria como técnica independiente.

Destacar la especificidad del método ya que ningún paciente del grupo control presento AM ni imágenes cistoscópicas que sugirieran la presencia de lesiones vesicales. Diferentes trabajos consultados presentan valores similares ${ }^{13,20,50}$.

Varios estudios previos describen la probable presencia de 2 o más genes supresores de tumores (TSG) en 9p21-p22 además del gen CDKN2A que está implicado en melanoma. Dada la gran cantidad de genes potencialmente implicados en los complejos procesos de iniciación tumoral, escape de la apoptosis, y metástasis, es posible que varios TSG involucrados en más de un tipo de tumor residan en una misma región genómica. La región de LOH estudiada por nosotros comprende además de un TSG ya descrito, el gen CDKN2A, implicado en melanoma, dos posibles TSG uno centromérico y otro telomérico respecto de CDKN2A. Estas regiones están implicadas en cáncer de vejiga, adenoma de pituitaria, cáncer de pulmón de células no pequeñas (NSCLC) cáncer de pulmón de células pequeñas (SCLC) y carcinoma de células escamosas de cuello y cabeza (HNSCC). Es muy probable que alguno de estos genes esté envuelto en uno de los primeros pasos que conducen al cáncer de vejiga ${ }^{29,51}$.

\section{CONCLUSIONES}

Con el análisis de microsatélites en células tumorales del sedimento urinario demostramos mayor eficacia en el diagnóstico de tumores vesicales que la tradicional citología. Nuestros resultados demuestran el aporte que pueden realizar las técnicas de biología molecular al elevar los porcentajes de sensibilidad sobre todo en él diagnostico de tumores de bajo grado y estadio. La presencia de alteraciones en 9p21-22 en tumores superficiales quedo en evidencia al encontrar mayor números de $\mathrm{LOH}$ en este grupo de pacientes. El hallazgo de MSI en cromosoma 4, estadísticamente significativo en tumores superficiales, 
podría deberse a la presencia en esta región de alteraciones moleculares que den inicio a los procesos de tumorogénesis vesical. Estas alteraciones implicadas en los procesos de generación y desarrollo del cáncer vesical pueden ser observadas en células del sedimento urinario utilizando un método no invasivo como lo es el análisis de microsatélites en orina.

Con la batería de marcadores utilizados en este estudio, 4 marcadores microsatélites de cromosoma 9p21 y uno de cromosoma 4, fuimos capaces de detectar el $74 \%$ de todos los tumores estudiados con solo dos casos de falsos positivos. $\mathrm{El}$ conocimiento de las diferentes regiones y sus marcadores microsatélites implicadas en la génesis del cáncer vesical permitirá confeccionar un grupo de marcadores que serian de gran utilidad para él diagnostico del cáncer vesical aumentado la sensibilidad del método.

\section{REFERENCIAS}

1. GREENLEE RT, MURRIA T, BOLDEN $\mathrm{S}$ et al.: Cancer statistics, 2000. CA Cancer J Clin 2000; 50: 7-33.

2. MARTÍNEZ PIÑEIRO JA, MARTÍNEZ PIÑEIRO L.: Tumores vesicales. En Tratado de Urología. J.F. Jiménez Cruz, A.L. Rioja Sáez. JR Prous Editores 1993: 1151-1152.

3. HOLMANG S, HEDELIN H, ANDERSTROM C, JOHANSSON SL.: The relationship among multiple recurrences, progression and prognosis of patients with stages Ta and T1 transitional cell cancer of the bladder followed for at least 20 years. J Urol 1995; 153:1823-1826.

4. WOLF H, KAKIZOE T, SMITH PH et al.: Bladder tumors. Treated natural history. Prog Clin Biol Res 1986; 221: 223-255.

5. PAPANICOLAOU GN, MARSHALL VF.: Urine sediment smears as a diagnostic procedure in cancers of the urinary tract. Science 1945; 101: 519-520.

6. SARNACKI CT, MCCORMACK LJ, KISER WS, HAZARD JB, MC LOUGHLIN TC, BELOVICH DM et al.: Urinary cytology and the clinical diagnosis of the urinary tract malignancy: a clinicpathologic study of 1400 patients. $J$ Urol 1971; 106: 761-764.

7. GUTIÉRREZ BANOS JL, MARTÍN GARCÍA B, DE DIEGO RODRÍGUEZ E et al.: El BTA STAT en el seguimiento del cáncer vesical. Arch Esp de Urología 1999; 52: 856-861.

8. RODRÍGUEZ MARTÍNEZ JJ, ESCAF BARMADAH S, ALLENDE MT et al.: Cuantificación del antígeno tumoral vesical (BTA Trak) y su correlación con el grado y estadio del carcinoma vesical. Arch Esp de Urología 2000; 53: 1-6.

9. WEBER JL, MAY PE.: Abundant class of human DNA polymorphisms which can be typed using the polymerise chain reaction. Am J Hum Genet 1989; 44: 388-396.
10. WEISSENBACH J, GYAPAY G, DIB C et al.: A second - generation linkage map of the human genome. Nature 1992; 359: 794-801.

11. MAO L, LEE DJ, TOCKMAN MS, EROZAN YS, ASKIN F, SIDRANSKY D.: Microsatellite alterations as clonal markers for the detection of human cancer. Proc Natl Acad Sci 1994; 91: 9871-9875.

12. SELLI C, TRAVAGLINI M, DAL CANTO M, PIAZZINI M, FRONTERA S, BARTOLETTI R.: Microsatellite analysis in the detection of bladder carcinoma: a preliminary report. Acta Urol Ital 1999; 13: 117120 .

13. MAO L, SCHOENBERG MP, SCICCHITANO M et al.: Molecular detection of primary bladder cancer by microsatellite analysis. Science 1996; 271: 659662.

14. CHEN XQ, STROUN M, MAGNENAT JL et al.: Microsatellite alterations in plasma DNA of small cell lung cancer patients. Nat Med 1996; 2: 10331035.

15. FUJIWARA Y, CHI DD, WANG H et al.: Plasma DNA microsatellites as tumor - specific markers and indicators of tumor progresión in melanoma patients. Cancer Res 1999; 59: 1567-1571.

16. NAWROZ H, KOCH W, ANKER $\mathrm{P}$ et al.: Microsatellite alterations in serum DNA of head and neck cancer patients. Nat Med 1996; 2: 10351037.

17. HIBI K, ROBINSON CR, BOOKER $\mathrm{S}$ et al.: Molecular detection of genetic alterations in the serum of colorectal cancer patients. Cancer Res 1998; 58: 1405-1407.

18. STEINER G, SCHOENBERG MP, LINN JF et al.: Detection of bladder cancer recurrence by microsatellite analysis of urine. Nat Med 1997; 3: 621624.

19. CHRISTENSEN M, JENSEN MA, WOLF H, ORNTOFT T et al.: Pronounced microsatellite instability in transitional cell carcinomas from young patients with bladder cancer. Int $J$ Cancer 1998; 79: 396401.

20. MOURAH S, CUSSENOT O, VIMONT V et al.: Assessment of microsatellite instability in urine in the detection of transitional cell carcinoma of the bladder. Int $J$ Cancer 1998; 79: 629-633.

21. BONNAL C, RAVERY V, TOUBLANC M et al.: Absence of microsatellite instability in transitional cell carcinoma of the bladder. Urology 2000; 55: 287-291.

22. HABUCHI T, DEVLIN J, ELDER PA, KNOWLES MA.: Delailed deletion mapping of chromosome $9 p$ in the bladder cancer: evidence for two tumour suppressor loci. Oncogene 1995; 11: 1671-1674.

23. SHAW ME, KNOWLES MA.: Deletion mapping of chromosome 11 in carcinoma of the bladder. Genes Chromosomes Cancer 1995; 13: 1-8.

24. PRESTI JC Jr, REUTER VE, GALAN T, FAIR WR, CORDÓN-CARDO C.: Molecular genetic alterations in superficial and locally advanced human bladder cancer. Cancer Res 1991; 51: 5405-5409.

25. MIYAMOTO H, SHUIN T, IWASAKI Y, KUBOTA Y.: Retinoblastoma gene mutations in primary human bladder cancer. Brit J Cancer 1995; 71: 831-835. 
26. HABUCHI H, OGAWA O, KAKEHI Y et al.: Alleleic loss of chromosome $17 \mathrm{p}$ in urothelial cancer: strong association with invasive phenotype. J Urol 1992; 148: 1595-1599.

27. KNOWLES MA, SHAW ME, PROCTOR AJ.: Deletion mapping of chromosome 8 in cancers of the urinary bladder using restriction fragment length polymorphisms and microsatellite polymorphisms. Oncogene 1993; 8: 1357-1364.

28. POLASCIK TJ, CARINS P, CHANG YH, SCJOENBERG MP, SIDRANSKY D.: Distinct regions of allelic loss on chromosome 4 in human primary bladder carcinoma. Cancer Res 1995; 55: 5396-5399.

29. Pollock PM et al.: Evidence for three tumor suppressor loci on chromosome 9p involved in melanoma development. Cancer Research 2001; 61: 11541161.

30. BROWN F.: Examen citológico de la orina. En "Cáncer Superficial de Vejiga": nuevas estrategias diagnósticas y terapéuticas. Clínicas de Urología de Norteamérica 2000; 1.

31. VOGELSTEIN B, FEARRON ER, KERN SE et al.: Allelotype of colorectal carcinomas. Science 1989; 244: 207-211.

32. THORSTENSEN L, QVIST H, NESLAND JM et al.: Allelotype profiles of local recurrences and distant metastases from colorectal -cancer patients. Int $J$ Cancer 1996; 69: 452-456.

33. LININGER R, FUJJI H, MAN YG et al.: Comparison of loss heterozygosity in primary and recurrent ductal carcinoma in situ of the breast. Mol Pathol 1998; 11: 1151-1159.

34. WALSH T, CHAPPELL SA, SHAW JA et al.: Microsatellite instability in ductal carcinoma in situ of the breast. J Pathol 1998; 185: 18-24.

35. TSUCHIYA E, NAKAMURA Y, WENG SY et al.: Allelotype of non-small cell lung carcinoma - comparison between loss heterozygosity in squamous carcinoma and adenocarcinoma. Cancer Res 1992; 52: $2478-2481$.

36. SHISEKI M, KOHNO T, ADACHI $J$ et al.: Comparative allelotype of early and advanced stage non-small cell lung carcinoma. Genes Chromosome Cancer 1996; 17: 71-77.

37. NAWROZ H, VAN DER RIET P, HRUBAN RH et al.: Allelotype of head and neck squamous cell carcinoma. Cancer Res 1994; 54: 1152-1155.

38. MORITA R, ISHIKAWA J, TSUTSUMI M et al.: Allelopype of renal cell carcinoma. Cancer Res 1991; 51: 820-823.

39. HEALY E, BELGAID CE, TAKATA $M$ et al.: Allelopyes of cutaneous melanoma and benign melanocytic nevi. Cancer Res 1996; 56: 589-593.

40. PARRELLA P, SIDRANSKY D, MERBS SL.: Allelopype of posterior uveal melanoma: implications for a fiburcated tumor progression. Cancer Res 1999; 59: 3032-3037.
41. SEPIRA D, PARRELLA P, GALLUCCI M et al.: Sensitive detection of transitional cell carcinoma of the bladder by microsatellite analysis of cell exfoliatedin urine. Int $J$ Cancer 2001; 95: 364-369.

42. NEVES M, CIOFU C, LAROUSSERIE $F$ et al.: Prospective evaluation of genetic abnormalities and telomerase expression in exfoliated urinary cells for bladder cancer detection. J Urol 2002; 167: 12761281.

43. SOURVINOS G, KAZANIS I, DELAKAS D, CRADINIS A, SPANDIDOS D.: Genetic detection of bladder cancer by microsatellite análisis of p16, RB1 and p53 tumor supresor genes. J Urol 2001; 165: 249-252.

44. BERGER AP, PARSON W, STENZL A, STEINER H, BARTSCH G, KLOCKER H.: Microsatellite alterations in human bladder cancer: detection of tumor cells in urine sediment and tumor tissue. European Urology 2002; 41: 532-539.

45. VON KNOBLOCH R, HEGELE A, BRANDT $\mathrm{H}$, OLBERT P, HEIDENREICH A, HOFMANN R.: Serun DNA and urine DNA. Alterations of urinary transitional cell bladder carcinoma detected by fluorescent microsatellite analysis. Int J Cancer 2001; 94: 67-72.

46. UTTING M, WERNER W, MULLER G, SCHUBERT J, JUNKER K.: A Possible noninvasive method for the detection of bladder cancer in patients: microsatellite analysis of free DNA in urine and blood. Ann NY Acad Sci 2001; 945: 31-35.

47. UTTING M, MULLER G, WERNER W et al.: Detection of tumor genetic alterations of bladder carcinomas in body fluids depends on sample treatment before DNA isolation. Ann NY Acad Sci 2000; 906: 67-71.

48. VAN RHIFN B, LURKIN I, KIRKELS W, VAN DER KWAST T, ZWARTHOFF E.: Microsatellite análisis DNA test in urine completes with cystoscopy in follow - up of superficial bladder carcinoma. Cancer 2001; 92: 768-775.

49. SHIBATA D, NAVIDI W, SALOVAARA R, LI ZH, AALTONEN AL.: Somatic microsatellite as molecular tumor clocks. Nature (Med) 1996; 2: 676-681.

50. LOTAN Y, ROEHRBORN CG.: Sensitivity and specificity of commonly available bladder tumor markers versus cytology: results of a comprehensive literature review an meta-analyses. J Urol 2001; 165: [abstract 680].

51. RUIZ A, PUIG S, LYNCH M, CASTEL T, ESTIVILL $\mathrm{X}$.: Retention of the CDKN2A locus and low prequency of point mutations in primary and metastatic cutaneous malignant melanoma. Int $J$ Cancer 1998; 76: 312-316.

Dr. R. Molina Burgos

C/ Sta. Cruz de la Zarza, 1 - 4º, p. 38

46021 Valencia

(Trabajo recibido el 21 abril de 2003) 